\title{
Briquetagem de resíduos de cafeeiro conduzido no sistema safra zero
}

\section{Briquetting of wastes from coffee plants conducted in zero harvest system}

\author{
Oberdan Everton Zerbinatti ${ }^{1}$; Adriano Bortolotti da Silva ${ }^{2 *}$; \\ Ademir José Pereira ${ }^{3}$; José Messias Miranda ${ }^{2}$
}

\begin{abstract}
Resumo
O processo de briquetagem consiste na densificação de resíduos lignocelulósicos em biocombustível sólido, de alto poder calorífico, denominado briquete. A cultura do café, importante commodity brasileira, produz resíduos culturais em quantidades diferentes dependendo dos tratos culturais. $\mathrm{O}$ Sistema Safra Zero de condução da lavoura é baseado em podas dos ramos plagiotrópicos em anos alternados, possibilitando a concentração da produção e evitando a bianualidade de produção cafeeiro. O presente trabalho teve por objetivo verificar a viabilidade de produção de briquetes a partir do emprego dos restos culturais obtidos no sistema safra zero. Os tratamentos consistiram da briquetagem de: 1) casca de café, 2) mistura de galhos e folhas; 3) $25 \%$ de casca de café $+75 \%$ de galhos e folhas; 4) $75 \%$ casca de café $+25 \%$ de galhos e folhas; 5) $50 \%$ casca de café $+50 \%$ de galhos e folhas; 6 ) $40 \%$ casca de café $+60 \%$ de galhos e folhas. Todas as misturas foram realizadas na base de $\mathrm{v} / \mathrm{v}$, sendo moídas gerando partículas de $5 \mathrm{a} 10 \mathrm{~mm}$, que ao atingirem $12 \%$ de umidade, foram processados na forma de briquetes. O teor de carbono dos briquetes produzidos variou de 41,85 a $43,84 \%$ e o de enxofre ficou abaixo de $0,1 \%$. O poder calorífico dos briquetes produzidos variou de $3.359,4$ a $4.028,3 \mathrm{kcal} \mathrm{kg}^{-1} \mathrm{e}$ as cinzas ficaram abaixo de $6 \%$. O uso de casca de café ou galhos e folhas de forma isolada, bem como a mistura de casca de café com $50 \%$ ou mais de galhos folhas permitem a produção de briquetes com pode calorífico superior (PCS) em torno dos $4.000 \mathrm{Kcal} \mathrm{kg}^{-1}$, que se encontra dentro dos parâmetros de qualidade. A briquetagem de resíduos da lavoura cafeeira é viável e sustentável energeticamente.
\end{abstract}

Palavras-chave: Biomassa, energia sustentável, densificação, resíduos lignocelulósicos

\begin{abstract}
The briquetting process consists of lignocellulosic residues densification in solid biofuel with high calorific value denominated briquette. Coffee crop is one of the most important Brazilian commodities and according to the cultural practices produces plant residues in different amounts. The zero harvest system in coffee crop is based in pruning of plagiotropic branches in alternated years to make possible to concentrate the harvest and to avoid coffee biannual production. The aim of the present work was to verify the viability of briquette production using the biomass waste obtained by zero harvest system. The treatments were composed of briquetting process: 1) coffee rind; 2) mixture of branches and leaves; 3) $25 \%$ of coffee rind $+75 \%$ of branches and leaves; 4) $75 \%$ of coffee rind $+25 \%$ of branches and
\end{abstract}

\footnotetext{
${ }^{1}$ Eng $^{\circ}$ Agr $^{\circ}$, Discente de Mestrado Profissional em Sistemas de Produção na Agropecuária, Universidade José do Rosário Vellano, UNIFENAS, Alfenas, MG. E-mail: oberdanzerbinati@yahoo.com.br

${ }^{2}$ Eng $^{\text {os }}$ Agros $^{\text {, }}$ Profs. Drs. em Produção Vegetal, Universidade José do Rosário Vellano, UNIFENAS, Alfenas, MG. E-mail: adriano. silva@unifenas.br; jose.miranda@unifenas.br

3 Prof. Dr. do Instituto Federal de Educação, Ciência e Tecnologia Sul de Minas Gerais, IFSULDEMINAS, Campus Inconfidentes, Inconfidentes, MG. E-mail: ademir.pereira@ifs.ifsuldeminas.edu.br

* Autor para correspondência
} 
leaves; 5) $50 \%$ of coffee rind $+50 \%$ of branches and leaves; 6) $40 \%$ of coffee rind $+60 \%$ of branches and leaves. The mixtures were realized in $\mathrm{v} / \mathrm{v}$ base, milled to produce $5-10 \mathrm{~mm}$ particles and were briqueted with $12 \%$ of humidity. The C-teor of briquettes produced ranged from 41.85 to $43.84 \%$ and sulphur teor was below $0.1 \%$. The calorific value of briquettes produced ranged from 3,359 to 4, 028 $\mathrm{Kcal} / \mathrm{kg}$ and the ashes were below $6 \%$. The isolated use of coffee rind or branches and leaves, as well the mixtures of coffee rind with $50 \%$ or more of branches and leaves allow the production of briquettes with calorific value around $4,000 \mathrm{Kcal} / \mathrm{kg}$ which is within the quality parameters. The briquetting of coffee crop wastes is viable and sustainable energetically.

Key words: Biomass, densification, sustainable energetic, lignocellulosic residues

\section{Introdução}

O Brasil é um país eminentemente agrícola. A cada ano são gerados aproximadamente 330 milhões de toneladas métricas $(\mathrm{Mg})$ de resíduos ou coprodutos lignocelulósicos no país. Estes resíduos freqüentemente não são usados como fonte de energia (FELFLI et al., 2011), devido a alta umidade, forma irregular, tamanho variado e baixa densidade, o que dificulta muito seu manuseio, armazenamento e transporte, e, portanto, a sua utilização na forma original (KALIYAN; MOREY, 2009). A fabricação de briquetes, a partir destes resíduos, é uma alternativa viável como fonte de energia renovável e ainda possibilita a conservação das florestas e da biodiversidade (RIGHELATO; SPRACKLEN, 2007; SCHARLEMANN; LAURANCE, 2008).

A briquetagem é um processo de aproveitamento de resíduos ou coprodutos lignocelulósicos. Esse processo consiste em densificar tal biomassa, gerando mecanicamente um aquecimento, que provoca a liquefação da lignina presente no material vegetal, que atua como agente aglomerante, dispensando a adição de outro agente aglomerante (ALBUQUERQUE; ANDRADE, 1997). Através desse processo subprodutos de beneficiamento de sistemas agroflorestais, agroindustriais e finos de carvão convertem-se em um material de maior valor comercial que é o briquete (ANTUNES, 1982).

A densificação (peletização e briquetagem) de biomassa, a partir da compactação de resíduos lignocelulósicos, usando pressão e temperatura (SILVA, 2007), aumenta o valor calorífico volumétrico e melhora o controle sobre os processos de combustão (GRANADA et al., 2002).
Quirino (1991) e Quirino et al. (2004) afirmaram que a densificação de resíduos pode proporcionar um aumento de cinco vezes ou mais na energia que o volume de resíduo que lhe deram origem, levandose em consideração a densidade a granel e o poder calorífico dos mesmos, transformando resíduos de baixa densidade em um produto densificado e com alto potencial energético. Além disso, a briquetagem permite a obtenção de um produto não só compactato, porém com forma, tamanho e parametros mecânicos adequados, bem como resistente ao apodrecimento ou a degradação devido a liginia que impermeabiliza a superfície do briquete, o que facilita o transporte e o armazenamento (RODRIGUES, 2010).

Resíduos ou coprodutos lignocelulósicos que foram originados em processos produtivos e não tem aplicação, podem ser utilizados como matériaprima na geração de calor ou eletricidade em grupos geradores ou usinas termelétricas (QUIRINO, 2003).

O uso de resíduos de biomassa vegetal para fins energéticos é já uma realidade. No entanto, a fim de garantir a adequação e recomendar um determinado material como sendo um gerador de energia é necessário caracterizar o material através de análise química e determinar seu valor calorífico (PAULA et al., 2011), bem como a mistura de materiais visando a produção de briquetes de alta qualidade. $\mathrm{O}$ briquete apresenta dimensões de diâmetro variando entre 50 a $100 \mathrm{~mm}$ e comprimento entre 250 a 400 $\mathrm{mm}$, umidade de 7 a $12 \%$, poder calorífico superior (PCS) de 17-18 MJ/kg e o teor de cinzas pode variar de acordo com a matéria prima, devendo ser inferior a 4\% para não gerar corrosão do queimador e desgaste de equipamentos (DIAS et al., 2012). 
O Sistema Safra Zero de condução da lavoura cafeeira consiste em dois tipos de podas, ou seja, é feito o decote do cafeeiro a uma altura variando de 1,50 a 1,80 metros e o esqueletamento dos ramos plagiotrópicos nos quatro lados dos pés de café a uma distância de $20 \mathrm{~cm}$ do tronco, visando principalmente a possibilidade de renovar e aumentar o número de ramos produtivos por planta, com isto potencializando a safra posterior (MATIELO; GARCIA; ALMEIDA, 2007).

Neste contexto, o presente trabalho teve por objetivo realizar a caracterização física, química e energética de briquetes obtidos a partir do processo de densificação de galhos folhas gerados pelo sistema de cultivo Safra Zero, bem como a casca resultante do beneficiamento de café.

\section{Material e Métodos}

O trabalho foi realizado no Instituto Federal de Educação, Ciência e Tecnologia do Sul de Minas, Campus Inconfidentes (IFSULMINAS), e na Fazenda Mangará, localizada em Ouro Fino, Sul do Estado de Minas Gerais, a uma altitude média de $997 \mathrm{~m}$, latitude de $22^{\circ} 16^{\prime} 58^{\prime \prime} \mathrm{S}$ e longitude de $46^{\circ} 22^{\prime} 08^{\prime}$ 'O. Apresenta clima do tipo tropical de altitude $\mathrm{CWb}$, com temperatura máxima de $34^{\circ} \mathrm{C}$ e a mínima de $2^{\circ} \mathrm{C}$, sendo a precipitação média anual de 1.744,2 mm (INMET, 2012).

Foram utilizadas plantas de cafeeiro (Coffea arabica L.), cultivar Mundo novo, com 26 anos de idade, com população de 5.000 plantas $\mathrm{ha}^{-1}$, espaçamento de $2,5 \times 0,80 \mathrm{~m}$, cultivadas em solo classificado como Latossolo Vermelho eutrófico, conduzidas com as melhores práticas agronômicas e produzindo o típico café especial de exportação da região Sul de Minas Gerais. As plantas foram esqueletadas e decotadas em julho de 2011, gerando $36.000 \mathrm{~kg} \mathrm{ha}^{-1}$ de massa verde.

Após o recolhimento do material, os ramos com frutos foram levados para máquina PAPAGALHOS $^{\circledR}$ (Figura 1A), onde foram separados os galhos e folhas dos frutos. Posteriormente, o material vegetal (galhos e folhas) foi moído utilizando um triturador de milho da marca Nogueira ${ }^{\circledR}$ com peneira $10(10 \mathrm{~mm})$. Após a moagem, o material retornou ao galpão para o processo de secagem à temperatura ambiente, sendo monitorado até atingir a umidade média de $12 \%$, quando foram realizadas as misturas de acordo com os tratamentos sendo, posteriormente, realizada a briquetagem.

Figura 1. A) Máquina PAPA-GALHOS ${ }^{\circledR}$; B) Briquetadeira BIOMAX ${ }^{\circledR}$; C) Aspecto geral dos briquetes produzidos de acordo com os diferentes tratamentos: Galhos e folhas (1); Casca de café (2); 25\% casca de café $+75 \%$ galhos e folhas (3); $75 \%$ casca de café $+25 \%$ galhos e folhas (4) $50 \%$ casca de café $+50 \%$ galhos e folhas (5); $40 \%$ casca de café $+60 \%$ galhos e folhas (6).
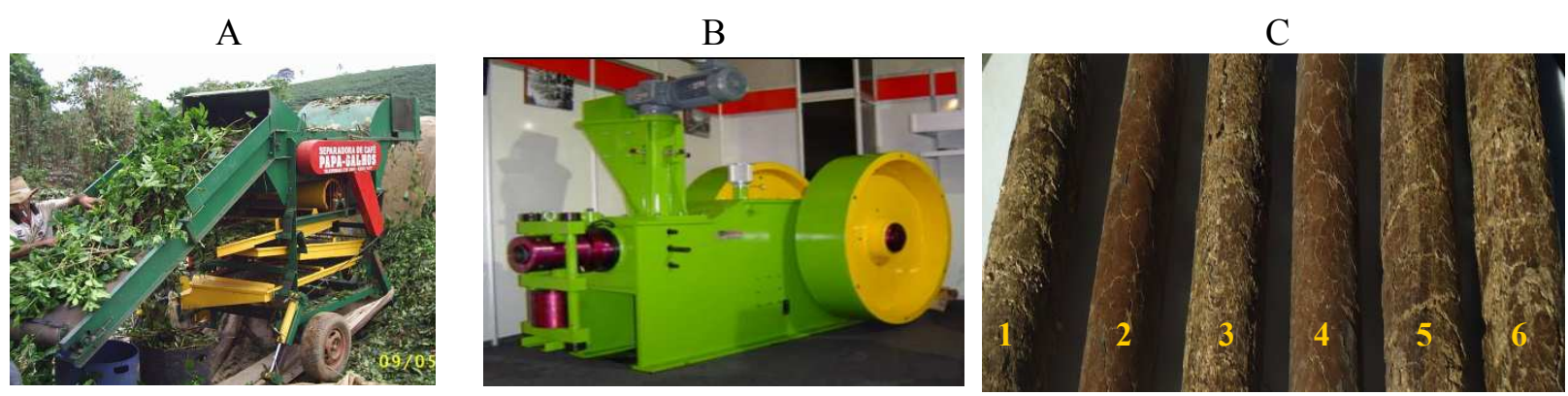

Fonte: Elaboração dos autores. 
Para a produção dos briquetes foram utilizados resíduos desde a colheita do café, no Sistema Safra Zero, bem como a casca advinda do processo de beneficiamento dos grãos de café. A briquetagem foi realizada utilizando galhos, folhas e palha de café em diferentes porcentagens de mistura em volume $(\mathrm{v} / \mathrm{v})$.

Os tratamentos consistiram da briquetagem de: 1) casca de café, 2) mistura de galhos e folhas; 3) $25 \%$ de casca de café $+75 \%$ de galhos e folhas; 4) $75 \%$ casca de café $+25 \%$ de galhos e folhas; 5 ) $50 \%$ casca de café $+50 \%$ de galhos e folhas; 6 ) $40 \%$ casca de café $+60 \%$ de galhos e folhas.

$\mathrm{Na}$ confecção dos briquetes foram utilizadas pressão de $150,04 \times 10^{5} \mathrm{~N} \mathrm{~m}^{-2}$ e temperatura entre 125 a $150^{\circ} \mathrm{C}$, faixa ideal para a plastificação da lignina, produzindo briquetes com comprimento de $34 \mathrm{~cm}$, diâmetro de 7,2 $\mathrm{cm}$ e peso aproximado de $1,506 \mathrm{Kg}$ (Figura 1C).

O processo de briquetagem foi realizado com material de granulometria máxima $10 \mathrm{~mm}$, com umidade entre 8 a $12 \%$, sendo processado em briquetadeira BIOMAX $^{\circledR}$ (Figura 1B), modelo B-75/190, que apresenta capacidade de produzir $4,5 \mathrm{~m}^{3}$ hora $^{-1}$ de briquetes, com um motor de $50 \mathrm{CV}$, volante com diâmetro de $1200 \mathrm{~mm}$. A compactação acontece através de golpes produzidos sobre a biomassa por meio de um pistão acionado através de dois volantes. $\mathrm{O}$ volante serve para armazenar energia cinética para sustentar o funcionamento contínuo do processo de briquetagem.

Para análise química elementar foram determinados, com analisador elementar, os teores de Carbono (C), Hidrogênio (H), Nitrogênio (N), Enxofre (S) e, por diferença, o teor de Oxigênio (O), conforme descrito por Bech, Jesen e Dam-Johansen (2009). Tal análise foi realizada em Analisador Elementar $^{\circledR}$, onde cada amostra foi retirada em separado, moída e peneirada. Posteriormente foram levadas à combustão uma massa conhecida da amostra à $925^{\circ} \mathrm{C}$, colocada em cápsula de estanho, utilizando gás hélio de arrasto para uma coluna de redução onde foram separados e determinados por cromatografia gasosa. A densidade dos briquetes foi realizada por análise gravimétrica, que se baseou no cálculo do volume pelo deslocamento de um fluído. Para este estudo, utilizou-se o mercúrio.

A análise química imediata foi fundamentada na Norma 8112 da Associação Brasileira de Normas Técnicas - ABNT (1983), para determinar os materiais voláteis e teor de cinzas. Teor de carbono fixo (CF) foi derivado da diferença (Equação 1).

$$
C F(\%)=100-M V(\%)-C I(\%)
$$

Onde:

$M V=$ Materiais voláteis

$C I=$ Cinzas

A análise para a determinação do poder calorífico superior (PCS) foi realizada em um calorímetro Parr $^{\circledR}$, seguindo a Norma 8633 da Associação Brasileira de Normas Técnicas, conforme descrito pela ABNT (1984). Para a determinação do poder calorífico inferior (PCI) foi empregada a equação 2.

$$
P C I=P C S-5,72 *(9 H+U)
$$

Onde:

$H=$ Hidrogênio $(\%)$

$U=$ Umidade em base seca (\%)

O delineamento utilizado foi o inteiramente casualizado (DIC), constando de seis tratamentos com 3 repetições. Os dados foram submetidos à análise de variância (ANAVA), utilizando-se do programa estatístico SISVAR (FERREIRA, 1999), sendo as médias comparadas pelo teste Scott \& Knott, a 5\% de probabilidade.

\section{Resultados e Discussão}

A análise de variância empregada $(p<0,05)$ para as variáveis em estudo possibilitou detectar 
efeitos significativos para o teor de nitrogênio e o carbono fixo, poder calorífico superior (PCS) e inferior (PCI), sendo que as demais variáveis ( $\mathrm{p}>0,05)$ não apresentaram efeito significativo. A Tabela 1 apresenta os resultados da análise química elementar. Observa-se que nos diferentes tratamentos a variação do teor de carbono ficou entre 41,85 e 43,84\%, o hidrogênio variou de 6,01 a $6,25 \%$, o nitrogênio variou de 0,95 a $1,91 \%$, o enxofre ficou abaixo de $0,1 \%$ e o oxigênio esteve na faixa de 45,10 a $49,90 \%$.
Os briquetes compostos somente por casca de café, ou com maior porcentagem de casca de café na sua constituição, apresentaram os menores teores de nitrogênio (Tabela 1). Huang et al. (2009) afirmaram que maior porcentual de nitrogênio presente nos briquetes, diminui a sua eficiência energética no processo de combustão, pois o nitrogênio compete com o carbono durante as reações de oxidação. Além disso, o teor está relacionado aos impactos ambientais e poluição do ar, em função da formação de óxidos de nitrogênio tóxicos e ácido nítrico (MUNALULA; MEINCKEN, 2009).

Tabela 1. Teor em porcentagem de massa de carbono $(\mathrm{C})$, hidrogênio $(\mathrm{H})$, nitrogênio $(\mathrm{N})$, enxofre $(\mathrm{S})$ e oxigênio $(\mathrm{O})$ nos briquetes produzidos a partir de diferentes proporções de casca de café, galhos e folhas.

\begin{tabular}{|c|c|c|c|c|c|}
\hline \multirow{2}{*}{ TRATAMENTOS } & \multicolumn{5}{|c|}{ ANÁLISE QUÍMICA ELEMENTAR (\%) } \\
\hline & $\mathrm{C}^{\text {(ns) }}$ & $\mathbf{H}^{\text {(ns) }}$ & $\mathbf{N}^{(*)}$ & $\mathbf{S}^{\text {(ns) }}$ & $\mathbf{O}^{\text {(ns) }}$ \\
\hline Galhos e folhas & $42,03^{\mathrm{a}}$ & $6,10^{\mathrm{a}}$ & $1,73^{\mathrm{a}}$ & $<0,1^{\mathrm{a}}$ & $45,10^{\mathrm{a}}$ \\
\hline casca de café & $43,64^{\mathrm{a}}$ & $6,01^{\mathrm{a}}$ & $0,95^{\mathrm{b}}$ & $<0,1^{\mathrm{a}}$ & $49,90^{\mathrm{a}}$ \\
\hline $25 \%$ casca de café $+75 \%$ galhos e folhas & $43,38^{\mathrm{a}}$ & $6,05^{\mathrm{a}}$ & $1,20^{\mathrm{b}}$ & $<0,1^{\mathrm{a}}$ & $46,33^{\mathrm{a}}$ \\
\hline $75 \%$ casca de café $+25 \%$ galhos e folhas & $43,49^{\mathrm{a}}$ & $6,01^{\mathrm{a}}$ & $0,96^{\mathrm{b}}$ & $<0,1^{\mathrm{a}}$ & $47,60^{\mathrm{a}}$ \\
\hline $50 \%$ casca de café $+50 \%$ galhos e folhas & $41,85^{\mathrm{a}}$ & $6,25^{\mathrm{a}}$ & $1,17^{\mathrm{b}}$ & $<0,1^{\mathrm{a}}$ & $47,10^{\mathrm{a}}$ \\
\hline $40 \%$ casca de café $+60 \%$ galhos e folhas & $42,50^{\mathrm{a}}$ & $6,11^{\mathrm{a}}$ & $1,91^{\mathrm{a}}$ & $<0,1^{\mathrm{a}}$ & $47,02^{\mathrm{a}}$ \\
\hline $\mathrm{CV}(\%)$ & 2,03 & 3,20 & 4,57 & 0,89 & 1,41 \\
\hline
\end{tabular}

Médias seguidas pela mesma letra minúscula na vertical não diferem entre si pelo Teste Scott \& Knott. * - significativo pelo teste $\mathrm{F}$ ao nível de $5 \%$ de probabilidade, ${ }^{\mathrm{ns}}$ - não significativo pelo teste $\mathrm{F}$ ao nível de $5 \%$ de probabilidade.

Fonte: Elaboração dos autores.

Brum et al. (2006), trabalhando com a casca de café, obtiveram, na análise elementar, 44\% de carbono, $5,40 \%$ de hidrogênio, $0,70 \%$ de nitrogênio e $49,90 \%$ de oxigênio. Estes resultados foram semelhantes aos encontrados no presente trabalho. Segundo Seye, Cortez e Gomez (2003), o teor de carbono do caule de cafeeiro gira em torno de 48,06 $\%$, diferente do presente trabalho, onde não foi observado nenhum teor acima de $43,84 \%$. Isto pode ter acontecido devido à mistura realizada de galhos, folhas e palha de café nos diferentes tratamentos, o que no final do processo reduziu o teor de carbono nos briquetes produzidos. Maiores teores de carbono e hidrogênio no briquete aumentam a eficiência na liberação de energia (DE SENA, 2005). Han e Kim (2008) relataram a quantidade de carbono armazenado na biomassa de plantas, comumente, é de aproximadamente $50 \%$ da biomassa em peso.

Para todos os tratamentos, o teor de enxofre obtido neste trabalho foi menor que $0,1 \%$ (Tabela 1), o que é positivo. Na produção de briquetes a partir de materiais lignocelulósicos (eucaliptos e/ ou outros), os valores de enxofre encontrados estão numa faixa superior entre 0,1 a $0,4 \%$, que juntamente com o nitrogênio contribuem relativamente pouco na liberação de energia de um combustível, além de ocasionarem poluição do ambiente e formação de chuva ácida após sua liberação na atmosfera durante a combustão (OBERNBERGER; BRUNNER; BÄRNTHALER, 2006; BILGEN; KAYGUSSUZ, 2008). Estudos mostram que tradicionalmente, o enxofre e as cinzas são considerados as principais 
impurezas dos combustíveis. Uma maior concentração de enxofre pode ocasionar a formação de componentes corrosivos nas caldeiras (TELMO; LOUSADA; MOREIRA, 2010).

As densidades dos briquetes variaram de 1,034 $\mathrm{g} \mathrm{cm}^{-3}$, com o tratamento composto pela mistura de $50 \%$ casca de café $+50 \%$ galhos e folhas (GF), até a densidade de $0,889 \mathrm{~g} \mathrm{~cm}^{-3}$, para o tratamento com $25 \%$ de casca de café $+75 \%$ GF (Tabela 2 ). Resultados semelhantes foram obtidos por Quirino
(1991) e Brito (1993), que encontraram densidades variando de 0,69 a $0,99 \mathrm{~g} \mathrm{~cm}^{-3}$ para briquetes produzidos com a mistura de serragem, carvão vegetal, nitrato, cinzas e aglomerantes. A densidade é importante para estocagem e transporte de material utilizado na geração de energia. Para a percentagem de umidade nos briquetes dos diferentes tratamentos, os resultados variaram de $11,86 \%$ para a mistura de $25 \%$ casca de café $+75 \%$ GF e de $17,61 \%$ de umidade para o tratamento composto por $75 \%$ casca de café $+25 \%$ GF (Tabela 2).

Tabela 2. Poder Calorífico Superior (PCS), Poder Calorífico Inferior (PCI), Umidade e Densidade nos briquetes produzidos a partir de diferentes proporções de casca de café, galhos e folhas.

\begin{tabular}{|c|c|c|c|c|}
\hline TRATAMENTOS & $\begin{array}{c}\begin{array}{c}\left.\text { PCS }{ }^{*}\right) \\
\left(\text { Kcal kg }^{-1}\right)\end{array}\end{array}$ & $\begin{array}{c}\left.\text { PCI }{ }^{*}\right) \\
\left(\text { Kcal kg }^{-1}\right)\end{array}$ & $\begin{array}{c}\text { UMIDADE } \\
(\%)\end{array}$ & $\begin{array}{c}\text { DENSIDADE (ns) } \\
\left(\mathrm{g} \mathrm{cm}^{-3}\right)\end{array}$ \\
\hline galhos e folhas & $3.973,7^{\mathrm{a}}$ & $3.795,2^{\mathrm{a}}$ & $12,57^{\mathrm{a}}$ & $0,958^{\mathrm{a}}$ \\
\hline casca de café & $4.005,9^{\mathrm{a}}$ & $3.822,1^{\mathrm{a}}$ & $13,28^{\mathrm{a}}$ & $1,030^{\mathrm{a}}$ \\
\hline $25 \%$ casca de café $+75 \%$ galhos e folhas & $4.028,3^{\mathrm{a}}$ & $3.846,7^{\mathrm{a}}$ & $11,86^{\mathrm{a}}$ & $0,889^{\mathrm{a}}$ \\
\hline $75 \%$ casca de café $+25 \%$ galhos e folhas & $3.359,4^{\mathrm{b}}$ & $3.176,8^{b}$ & $17,61^{\mathrm{a}}$ & $1,006^{\mathrm{a}}$ \\
\hline $50 \%$ casca de café $+50 \%$ galhos e folhas & $3.746,9^{\mathrm{a}}$ & $3.575,6^{\mathrm{a}}$ & $15,21^{\mathrm{a}}$ & $1,034^{\mathrm{a}}$ \\
\hline $40 \%$ casca de café $+60 \%$ galhos e folhas & $3.916,1^{\mathrm{a}}$ & $3.740,6^{\mathrm{a}}$ & $15,18^{\mathrm{a}}$ & $1,013^{\mathrm{a}}$ \\
\hline CV $(\%)$ & 5,36 & 6,23 & 1,40 & 1,71 \\
\hline
\end{tabular}

Médias seguidas pela mesma letra minúscula na vertical não diferem entre si pelo Teste Scott \& Knott. * - significativo pelo teste $\mathrm{F}$ ao nível de $5 \%$ de probabilidade, ${ }^{\text {ns }}$ - não significativo pelo teste $\mathrm{F}$ ao nível de $5 \%$ de probabilidade.

Fonte: Elaboração dos autores.

Reis et al. (2002) relataram que briquetes com teor de umidade superior a $15 \%$ podem comprometer a eficiência de sua combustão devido a relação negativa entre poder calorífico e umidade. Entretanto, para a compactação do resíduo é necessário teor de umidade num faixa estreita de 8 a $15 \%$ para a liquefação da lignina e conseqüentemente a ligação das partículas durante a compactação (CARVALHO; BRINCK, 2004; SHAW; KARUNAKARAN; TABIL, 2009). No presente trabalho, observou-se a tendência de aumento da umidade nos briquetes nos tratamentos que foram compostos por misturas com maior quantidade de casca de café (Tabela 2), o que levou, provavelmente, a uma diminuição do Poder Calorífico Superior (PCS) e Inferior (PCI), nestes tratamentos, principalmente, na combinação de $75 \%$ palha $+25 \%$ GF, que apresentou o menor poder calorífico (Tabela 2) e umidade superior a $15 \%$. Briquetes com teores de umidade em torno de $12 \%$ resultaram em maiores PCS e PCI (Tabela 2).

A média dos maiores valores PCS foi de 3.934,18 Kcal kg-1 e dos maiores PCI ficou em $3.756,03 \mathrm{Kcal} \mathrm{kg}^{-1}$ (Tabela 2). No presente trabalho, o tratamento composto somente por palha de café apresentou valores de 4.005,9 e 3.822,1 Kcal kg-1 para PCS e PCI, respectivamente. Estes valores foram inferiores aos observados por Paula et al. (2011), que encontraram para pergaminho de café valores de 4.441,74 $\mathrm{Kcal} \mathrm{kg}^{-1}$ para PCS e 4.017, 89 $\mathrm{Kcal} \mathrm{kg}^{-1}$ e para o caule de café o valor do PCS foi 4.544,00 Kcal kg-1 e PCI foi de 4.125,30 Kcal kg-1. Ainda para fins comparativos, a madeira apresentou poder calorífico de 4.000 a $4.800 \mathrm{Kcal} \mathrm{kg}^{-1}$ (BRITO, 1993), o bagaço de cana de açúcar apresentou 
4.139,0 Kcal kg-1 (SEYE; CORTEZ; GOMEZ, 2003) e para algumas espécies cultivadas como o arroz, milho, soja, feijão o PCS variou de 3. 812, 30 a 4.499,74 Kcal kg-1 e o PCI de 3.445, 05 a 4.021,70 Kcal kg-1 (PAULA et al., 2011).

As cinzas geradas na queima correspondem à porcentagem de material que não produz calor e pode afetar na contribuição energética do material.
Estes materiais utilizam a energia fornecida durante a queima para se manterem aquecidos (GONÇALVES; SARTORI; LEÃO, 2009). No presente trabalho o teor de cinzas ficou abaixo de 6, $15 \%$ (Tabela 3). Em resíduos florestais o teor de cinzas é de $2 \%$, conforme relataram Ayoub et al. (2006). Shaw e Karunakaram e Tabil (2009), trabalhando com palha trigo e álamo, observaram teor de cinzas de 4,02 e 0,54\% respectivamente.

Tabela 3. Teor de voláteis, carbono fixo e cinzas nos briquetes produzidos a partir de diferentes proporções de casca de café, galho e folhas.

\begin{tabular}{lccc}
\hline \multirow{2}{*}{ TRATAMENTOS } & \multicolumn{2}{c}{ ANÁLISE QUÍMICA IMEDIATA (\%) } \\
\cline { 2 - 4 } & Voláteis $^{(\text {(n) }}$ & Carbono fixo $^{(*)}$ & Cinzas $^{(\mathbf{n s})}$ \\
\hline galhos e folhas & $72,20^{\mathrm{a}}$ & $22,80^{\mathrm{a}}$ & $5,00^{\mathrm{a}}$ \\
casca de café & $78,00^{\mathrm{a}}$ & $17,67^{\mathrm{b}}$ & $4,33^{\mathrm{a}}$ \\
$25 \%$ casca de café + 75\%galhos e folhas & $80,57^{\mathrm{a}}$ & $14,62^{\mathrm{b}}$ & $4,81^{\mathrm{a}}$ \\
$75 \%$ casca de café + 25\%galhos e folhas & $87,17^{\mathrm{a}}$ & $7,93^{\mathrm{c}}$ & $4,90^{\mathrm{a}}$ \\
$50 \%$ casca de café $+50 \%$ galhos e folhas & $83,68^{\mathrm{a}}$ & $11,95^{\mathrm{c}}$ & $4,37^{\mathrm{a}}$ \\
$40 \%$ casca de café + 60\%galhos e folhas & $80,07^{\mathrm{a}}$ & $13,78^{\mathrm{b}}$ & $6,15^{\mathrm{a}}$ \\
\hline CV $(\%)$ & 6,67 & 5,04 & 5,92 \\
\hline
\end{tabular}

Médias seguidas pela mesma letra minúscula na vertical não diferem entre si pelo Teste Scott \& Knott. * - significativo pelo teste $\mathrm{F}$ ao nível de $5 \%$ de probabilidade, ${ }^{\text {ns }}$ - não significativo pelo teste $\mathrm{F}$ ao nível de $5 \%$ de probabilidade.

Fonte: Elaboração dos autores.

Os materiais voláteis encontrados na análise química imediata giraram em torno de 72 a $87 \%$ e o carbono fixo ficou entre 7,9 a $22,80 \%$ (Tabela 3). Rodrigues (2010) trabalhando com briquetes de finos de madeira em mistura com lodo encontrou $15 \%$ de carbono fixo e $90 \%$ de materiais voláteis na ausência de lodo. A mesma autora relatou ainda que o aumento das concentrações de lodo no briquete diminuiu a porcentagem de materiais voláteis e carbono fixo. Os materiais voláteis têm papel importante durante a ignição e as fases de combustão da biomassa (IVANOV; SUDAKOVA; KUZNETSOW, 2003; CORTEZ; LORA; GOMEZ, 2008).

O Poder Calorífico Superior (PCS) com Carbono Fixo (CF) apresenta uma relação positiva, ou seja, quanto maior o CF maior o PCS (Tabelas 2 e 3). $\mathrm{O}$ teor de Carbono Fixo tem influência direta no poder calorífico (DE SENA, 2005). Briquetes com maior densidade apresentam maior estabilidade e durabilidade; da mesma forma que briquetes com alta porcentagem de carbono fixo e poder calorífico tem baixo teor de cinzas e material volátil (SOTANNDE; OLUYEGE; ABAH, 2010).

$\mathrm{O}$ alto índice de carbono fixo é usado como referência porque se pressupõe que à medida que aumenta a quantidade de carbono fixo, a possibilidade de liberação de voláteis nocivos é menor. No entanto, deve-se ressaltar que os voláteis nocivos são gerados pelo baixo teor de carbono fixo ou pela combustão em condições inapropriadas, bem como pelo alto teor de umidade contido no combustível (SUAREZ; LUENGO, 2003). No presente trabalho maior teor de carbono fixo foi encontrado nos briquetes compostos somente por galhos e folhas (Tabela 3), houve a tendência de 
briquetes obtidos em misturas com o emprego de porcentual igual ou superior a $50 \%$ de galhos e folhas em combinação com casca de café apresentar maiores teores de carbono fixo (tabela 3), o que acarretou em aumento no PCS (Tabela 2).

O uso de casca de café ou galhos e folhas de forma isolada permitiram a produção de briquetes com poder calorífico superior (PCS) em torno dos $4.000 \mathrm{Kcal} \mathrm{kg}^{-1}$ (Tabela 2), os quais se encontram dentro dos parâmetros de qualidade. Os briquetes produzidos a partir da mistura de casca de café com galhos e folhas também apresentaram bom desempenho em relação ao PCS, exceto os briquetes obtidos pela combinação de $75 \%$ casca de café com $25 \%$ galhos e folhas, que apresentou menor teor de carbono fixo e alto teor de umidade (Tabelas 2 e 3), o que contribuiu para queda no PCS (Tabela 2).

\section{Conclusões}

Briquetes produzidos com resíduos advindos da cultura do café, cultivado em sistema de Safra Zero, apresentam características físico-químicas que demonstram ser uma fonte viável para geração de energia e aproveitamento de resíduos.

De acordo com resultados obtidos pode-se concluir que os briquetes apresentam baixos teores de enxofre e nitrogênio, o que reduz a emissão a emissão de poluentes atmosféricos (SOx e NOx). Briquetes produzidos com a casca de café, bem como galhos e folhas de forma isolada ou a mistura de casca de café com $50 \%$ ou mais de galhos folhas são recomendados como fonte energética para uso doméstico e industrial, tais como churrasqueiras, fornos de padarias ou pizzarias, caldeiras entre outros.

\section{Referências}

ALBUQUERQUE, C. E. C.; ANDRADE, A. M. Visão histórica e perspectiva futura. Floresta e Ambiente, Rio de Janeiro, v. 4, p. 104-109, 1997.
ANTUNES, R. C. Briquetagem de carvão vegetal. In: FUNDAÇÃO CENTRO TECNOLÓGICO DE MINAS GERAIS/CETEC. Carvão vegetal: destilação, propriedades e controle de qualidade. Belo Horizonte: CETEC, 1982. p. 197-206.

ASSOCIAÇÃO BRASILEIRA DE NORMAS TÉCNICAS - ABNT. NBR 8112: carvão vegetal: análise imediata. Rio de Janeiro, 1983. 6 p.

NBR 8633: carvão vegetal: determinação do poder calorífico. Rio de Janeiro, 1984. 13 p.

AYOUB, N., WANG, K.; SEKI, H.; NAKA, Y. Towards sustainable electricity production from Japanese forestry residues: supply chains scenarios and parameters estimation model. Journal of life cycle Assessments Japan, Tokyo, v. 2, n. 3, p. 212-221, 2006.

BECH, N.; JESEN, P. A.; DAM-JOHANSEN, K. Determining the elemental composition of fuel by bomb calorimetry and the inverse correlation of HHV with elemental composition. Biomass \& Bioenerg, Cambridge, v. 33, n. 3, p. 534-537, 2009.

BILGEN, S.; KAYGUSSUZ, K. The calculation of the chemical exergies of coal-based fuels by using the higher heating values. Applied Energy, London, v. 85, n. 8, p. 776-785, 2008.

BRITO, J. O. Expressão da produção florestal em unidades energéticas. In: CONGRESSO FLORESTAL PANAMERICANO, 1.; CONGRESSO FLORESTAL BRASILEIRO, 7., 1993, Curitiba. Anais... Curitiba: Sociedade Brasileira de Silvicultura, 1993, p. 280-282.

BRUM, S. S.; SILVA, V. L.; BIANCHI, M. L.; REIS, M. C. Caracterização química do resíduo da colheita de feijão (palha de feijão). In: CONGRESSO DOS PÓSGRADUANDOS DA UNIVERSIDADE FEDERAL DE LAVRAS, 15., 2006, Lavras, MG. Anais... Lavras: UFLA, 2006. CD-ROM.

CARVALHO, E. A.; BRINCK, V. Briquetagem. In: LUZ, A. B. da; SAMPAIO, J. A.; ALMEIDA, S. L. M. Tratamento de minérios. Rio de Janeiro: CETEM, 2004. p. 603-636.

CORTEZ, L. A. B.; LORA, E. S.; GOMEZ, E. O. Caracterização da biomassa. In: GOMEZ, E. O. Biomassa para energia. Campinas: UNICAMP, 2008. p. 31-62.

DE SENA, R. F. Avaliação da biomassa obtida pela otimização da flotação de efluentes da indústria de carnes para geração de energia. 2005. Dissertação (Mestrado em Engenharia Química) - Departamento de Engenharia Química e de Alimentos. Universidade Federal de Santa Catarina, Florianópolis. 
DIAS, M. C. S.; SOUZA, D. T.; BRAGA, M.; ONOYAMA, M. M.; MIRANDA, C. H. B.; BARBOSA, P. F. D.; ROCHA, J. D. Produção de briquetes a partir de resíduos agrícolas, agroindustriais e florestais. Brasília: Embrapa Agroenergia, 2012. 130 p. (Documentos, 13).

FELFLI, F. F.; MESA, P. J. M. M.; ROCHA, J. D.; FILIPPETTO, D.; LUENGO, C. A.; PIPPO, W. A. Biomass briquetting and its perspectives in Brazil. Biomass and Bioenergy, Cambridge, v. 35, n. 1, p. 236242, 2011.

FERREIRA, D. F. SISVAR - Sistema de analises estatísticas. Lavras: UFLA, 1999.

GONÇALVES, J. E.; SARTORI, M. M. P.; LEÃO, A. L. Energia de briquetes produzidos com rejeitos de resíduos sólidos urbanos e madeira de eucalypitus grandis. Revista brasileira de Engenharia Agrícola e Ambiental, Campina Grande, v. 13, n. 5, p. 657-661, 2009.

GRANADA, E.; LOPEZ GONZALEZ, L. M.; MIGUEZ, J. L.; MORAN, J. Fuel lignocellulosic briquuettes, die desingn and products study. Renewable Energy, Oxford, v. 27, n. 4, p. 561-573, 2002.

HAN, J.; KIM, H. The reduction and control technology of tar during biomass gasification/pyrolysis: an overview. Renewable Sustainable Energy Reviews, Golden, v. 12, n. 2, p. 397-416, 2008.

HUANG, C.; HAN, L.; YANG, Z.; LIU, X. Ultimate analysis and heating value prediction of straw by near infrared spectroscopy. Waste Management, Oxford, v. 29, n. 6, p. 1793-1797, 2009.

INSTITUTO NACIONAL DE METEOROLOGIA INMET. Banco de dados meteorólogicos para ensino e pesquisa (BDMEP). Brasília: INMET, 2012. Disponível em: <http://www.inmet.gov.br/portal/http://www.inmet. gov.br/portal/>. Acesso em: 10 jul. 2012.

IVANOV, I. P.; SUDAKOVA, I. G.; KUZNETSOW, B. Manufacture of briquetted and granulated fuel from lignite with biobinders and heated die. Chemistry for Sustainable Development, Novosibirsk, v. 11, p. 847$852,2003$.

KALIYAN, K.; MOREY, R. V. Factors affecting strength and durability of densified biomass products. Biomass and Bioenergy, Oxford, v. 33, n. 3, p. 337-359, 2009.

MATIELO, J. B.; GARCIA, A. W. R.; ALMEIDA, S. R. A poda em cafezais. Revista Brasileira de Tecnologia Cafeeira: Coffea, Lavras, v. 4, n. 11, p. 33-34, 2007.

MUNALULA, F.; MEINCKEN, M. An evaluation of Souith African fuelwood with regards to calorific value and environmental impact. Biomass \& Bioenergy, Cambrigde, v. 33, n. 3, p. 415-420, 2009.
OBERNBERGER, I.; BRUNNER, T.; BÄRNTHALER, G. Chemical properties of solid biofuels - significance and impact. Biomass \& Bioenergy, Cambrigde, v. 30, n. 11, p. 973-982, 2006.

PAULA, L. E. R. de; TRUGILHO, P. F.; NAPOLI, A.; BIANCHI, M. L. Characterization of residues from plant biomass for use in energy generation. Cerne, Lavras, v. 17, n. 2, p. 237-246, 2011.

QUIRINO, W. F. Briquetagem de resíduos lignocelulósico. Circular Técnica do Laboratório de Produtos Florestais-LPF, Brasília, v. 1, n. 2, p. 69-80, 1991.

QUIRINO, W. F. Utilização energética de resíduos vegetais. Brasília: LPF/IBAMA, 2003. 14 p.

QUIRINO, W. F.; VALE, A. T. do; ANDRADE, A. P. A. de; ABREU, V. L. S.; AZEVEDO, A. C. dos S. Poder calorífico da madeira e de resíduos lignocelulósicos. Biomassa e Energia, Viçosa, v. 1, n. 2, p. 173-182, 2004.

REIS, B. de O.; SILVA, I. T. da; SILVA, M. O. da; ROCHA, B. R. P. da. Produção de briquetes energéticos a partir de caroço de açaí. In: ENCONTRO ENERGÉTICO MEIO RURAL, 4., 2002, Campinas. Anais... Campinas: UNICAMP, 2002. CD-ROM.

RIGHELATO, R.; SPRACKLEN, D. V. Carbon mitigation by biofuels or saving and restoring Forest? Science, Washington, v. 317, n. 5840, p. 902-907, 2007.

RODRIGUES, V. A. J. Valorização energética de lodo biológico da indústria de polpa celulósica através da briquetagem. 2010. Dissertação (Mestrado em Ciência Florestal) - Universidade Federal de Viçosa, Viçosa, MG.

SCHARLEMANN, J. P. W.; LAURENCE, W. F. How green are biofuels? Science, Washington, v. 319, n. 5859, p. 34-44, 2008.

SEYE, O.; CORTEZ, L. A. B.; GOMEZ, E. O. Estudo cinético de biomassa a partir de resultados termogrravimétricos. In: ENCONTRO ENERGÉTICO MEIO RURAL, 5., 2003, Campinas. Anais... Campinas. UNICAMP, 2003. CD-ROM.

SHAW, M. D.; KARUNAKARAN, L. G.; TABIL, A. Physicochemical characteristics of densified untreated and steam exploded poplar wood and wheat straw grinds. Biosystems Engineering, England, v. 103, n. 2, p. 198207, 2009.

SILVA, C. A. da. Estudo técnico-econômico da compactação de resíduos madeireiros para fins energéticos. 2007. Dissertação (Mestrado em Planejamentos de sistemas Energéticos) - Faculdade de Engenharia Mecânica. Universidade Estadual de Campinas, Campinas. 
SOTANNDE, O. A.; OLUYEGE, A. O.; ABAH, G. B. TELMO, C.; LOUSADA, J.; MOREIRA, N. Proximate Physical and combustion properties of briquettes from sawdust of Azadirachta indica. Journal of Forestry Reserch, Harbin, v. 21, n. 1, p. 63-67, 2010.

SUAREZ, J. A.; LUENGO, C. A. Coffee husk briquettes: analysis, backwards stepwise regression between gross calorific value, ultimate and chemical analysis of wood. Bioresource Technology, New York, v. 101, n. 11, p. a new renewable energy source. Energy Source, London, v. 25, n. 10 , p. 961-967, 2003. 3808-3815, 2010. 